\title{
Transmitting Conditions of Cable Tree
}

\author{
Vaclav Krepelka ${ }^{1}$ and Miloslav Filka ${ }^{2}$ \\ ${ }^{1}$ FITCE CZ, Mahenova 2, Brno \\ vaclav.krepelka@iol.cz \\ ${ }^{2}$ Department of Telecommunications, Purkynova 118, Brno \\ Faculty of Electrical Engineering and Communication \\ Brno University of Technology \\ filka@feec.vutbr.cz
}

\begin{abstract}
Research and development of Spectrum Administration for metallic access networks analyzed possibilities of matrix representation for transmission circumstances. Some interesting remarks were gained as a side output of this work.
\end{abstract}

Keywords: Cable tree, signal spectrum function, noise spectrum function, transmitting function, PWD.

\section{Cable tree}

Key term Cable tree is defined in the last mile topology. Cable tree itself consists of sequence coupled cable sections concentrated in site of Main distribution frame in common trunk cable, see Fig. 1.

Note: Cable tree may be equipped by optical network units ONU, where optical signal is transformed into electrical and vice versa respectively; and which is in ONU-MDF received or transmitted into/from pairs of tided metallic cable.

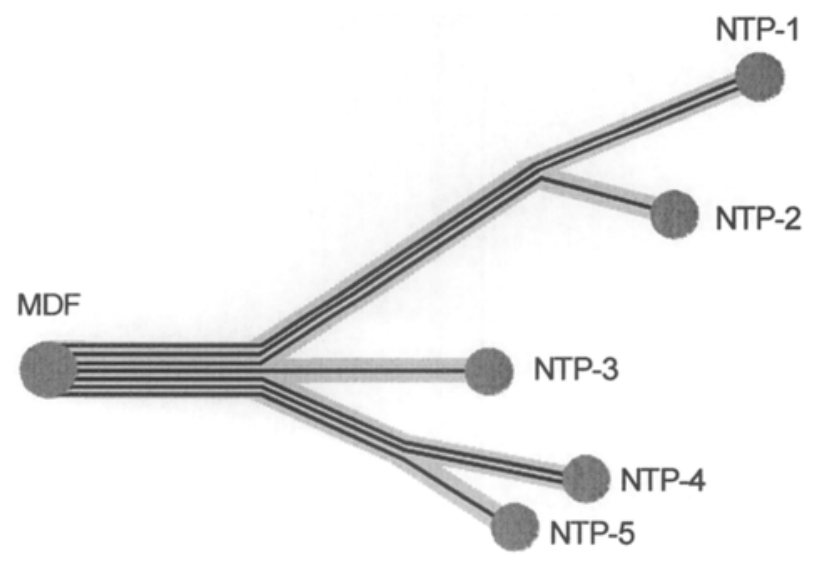

Fig. 1. Basic architecture of cable tree

Please use the following format when citing this chapter:

Krepelka, V., Filka, M., 2007, in IFIP International Federation for Information Processing, Volume 245, Personal

Wireless Communications, eds. Simak, B., Bestak, R., Kozowska, E., (Boston: Springer), pp. 610-615. 


\section{Analytical Performance of Useful Signals \& Disturbing Noises}

Circumstances of mutual influences upon symmetric metallic cable are performed in Fig. 2:

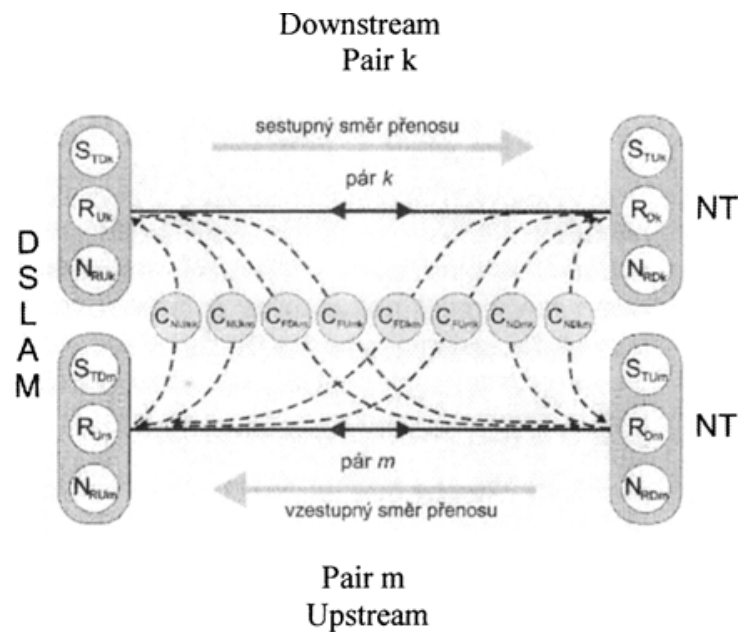

Fig. 2: Signals a Couplings between two Pairs

The simplest generalizable case of bidirectional transmission alongside two symmetric pairs is performed here. These pairs are terminated in exchange site in v DSLAM, in subscriber site in two network terminations. Three transmission characteristics belong to each of four here performed terminating points:

- Transmitted spectrum of signals $\boldsymbol{S}(\boldsymbol{\omega})$ or maybe $\boldsymbol{S}(\boldsymbol{f})$

- Non wanted disturbing noise $\quad N(\omega)$

- Receiver sensitivity $\boldsymbol{R}(\boldsymbol{\omega})$

as well as inter pair crosstalk couplings

- Near end crosstalk (NEXT) $\quad C_{N}(\omega)$

- Far end crosstalk (FEXT) $\quad C_{F}(\boldsymbol{\omega})$

Indexes $\boldsymbol{k}$ and $\boldsymbol{m}$ define mutual position of pairs;

indexes $\boldsymbol{D}$ and $\boldsymbol{U}$ discriminate downstream and upstream transmission directions; indexes $\boldsymbol{T}$ and $\boldsymbol{R}$ assign appropriate characteristics to transmission and receive of signal.

Transmitting circumstances in site of subscriber termination, in Fig. 1 pointed NT are expressed by following equation:

$$
S_{R D k}(\omega)=T_{C T k}(\omega) S_{T D k}(\omega)
$$

Equally in site of exchange termination $(D S L A M)$ is valid:

$$
S_{R U k}(\omega)=T_{C T k}(\omega) S_{T U k}(\omega)
$$

where

$T_{C T}$ is a vector of cable tree transmitting functions 
Noise circumstances in site of NT may be described by following equation:

$$
N_{R D k}(\omega)=S_{T D m}(\omega) C_{F D m k}(\omega)+S_{T U m}(\omega) C_{N D m k}(\omega)
$$

as well as dually in site exchange termination DSLAM

$$
N_{R U k}(\Phi)=S_{T D m}(\infty) C_{N U m k}(\omega)+S_{T U m}(\infty) C_{F U m k}(\infty)
$$

Such circumstances are only exceptional in reality; maybe only in last cable section led from subscriber cabinet supposing one-quad subscriber cable and both pairs exploited by broadband service. All couplings in real cable tree are multiplied and combined many times. We are able to express these circumstances by matrixes. These matrixes will be of equal order to number of pairs in trunk cable of cable tree. This number of pairs may reach hundreds as well as exceed thousand in metropolitan networks. Composition of such matrix exceeds real possibility of calculation.

Note: Variable $\boldsymbol{\omega}$ will be again left out in following text

The equation $1 \mathbf{a}$ is able to be performed by product of diagonal matrix:

$$
\left[\begin{array}{cccc}
S_{R D 1} & 0 & 0 & 0 \\
0 & S_{R D 2} & \ldots & \ldots \\
\ldots & \ldots & \ldots & 0 \\
0 & \ldots & 0 & S_{R D p}
\end{array}\right]=\left[\begin{array}{cccc}
T_{C T 1} & 0 & \ldots & 0 \\
0 & T_{C T p} & \ldots & \ldots \\
\ldots & \ldots & \ldots & 0 \\
0 & \ldots & 0 & T_{C T p}
\end{array}\right] *\left[\begin{array}{cccc}
S_{T D 1} & 0 & \ldots & 0 \\
0 & S_{T D 2} & \ldots & \ldots \\
\ldots & \ldots & \ldots & 0 \\
0 & 0 & 0 & S_{T D p}
\end{array}\right]
$$

Equally equation $1 b$ :

$$
\left[\begin{array}{cccc}
S_{R U 1} & 0 & 0 & 0 \\
0 & S_{R U 2} & \ldots & \ldots \\
\ldots & \ldots & \ldots & 0 \\
0 & \ldots & 0 & S_{R U p}
\end{array}\right]=\left[\begin{array}{cccc}
T_{C T 1} & 0 & \ldots & 0 \\
0 & T_{C T_{p}} & \ldots & \ldots \\
\ldots & \ldots & \ldots & 0 \\
0 & \ldots & 0 & T_{C T p}
\end{array}\right] *\left[\begin{array}{cccc}
S_{T U 1} & 0 & \ldots & 0 \\
0 & S_{T U 2} & \ldots & \ldots \\
\ldots & \ldots & \ldots & 0 \\
0 & 0 & 0 & S_{T U p}
\end{array}\right]
$$

The equation 2 a performing noise circumstances by matrixes of $p^{\text {th }}$ order for $p$ pairs of cable tree trunk cable as a product of left diagonal signal matrix multiplied by right crosstalk matrix with zero diagonal. Resulting noise matrix is again with zero diagonal in accordance with multiplying rule for matrixes. 
$\left[\begin{array}{cccc}0 & N_{R D 12} & \cdots & N_{R D 1 p} \\ N_{R D 21} & 0 & \cdots & \cdots \\ \cdots & \cdots & 0 & N_{R D(p-1) p} \\ N_{R D p 1} & \cdots & N_{R D p(p-1)} & 0\end{array}\right]=$

$\left[\begin{array}{cccc}S_{T D 1} & 0 & \ldots & 0 \\ 0 & S_{T D 2} & \cdots & \cdots \\ \cdots & \cdots & \cdots & 0 \\ 0 & 0 & 0 & S_{T D p}\end{array}\right] *\left[\begin{array}{cccc}0 & G_{F D 12} & \ldots & G_{F D 1 p} \\ G_{F D 21} & 0 & \ldots & \ldots \\ \ldots & \ldots & 0 & G_{F D(p-1) p} \\ G_{F D p 1} & \ldots & G_{F D p(p-1)} & 0\end{array}\right]+$

$+\left[\begin{array}{cccc}S_{T U 1} & 0 & \ldots & 0 \\ 0 & S_{T U 2} & \ldots & \ldots \\ \ldots & \ldots & \ldots & 0 \\ 0 & 0 & 0 & S_{T U p}\end{array}\right] *\left[\begin{array}{cccc}0 & G_{N U 12} & \ldots & G_{N U 1 p} \\ G_{N U 21} & 0 & \ldots & \ldots \\ \ldots & \ldots & 0 & G_{N U(p-1) p} \\ G_{N U p 1} & \ldots & G_{N U p(p-1)} & 0\end{array}\right]$

The equation $2 \mathrm{~b}$ may be performed dually as follows:

$\left[\begin{array}{cccc}0 & N_{R U 12} & \cdots & N_{R U 1 p} \\ N_{R U 21} & 0 & \cdots & \cdots \\ \cdots & \cdots & 0 & N_{R U(p-1) p} \\ N_{R U p 1} & \cdots & N_{R U p(p-1)} & 0\end{array}\right]=$

$\left[\begin{array}{cccc}S_{T D 1} & 0 & \ldots & 0 \\ 0 & S_{T D 2} & \ldots & \ldots \\ \ldots & \ldots & \ldots & 0 \\ 0 & 0 & 0 & S_{T D p}\end{array}\right] *\left[\begin{array}{cccc}0 & G_{N U 12} & \ldots & G_{N U 1 p} \\ G_{N U 21} & 0 & \ldots & \ldots \\ \ldots & \ldots & 0 & G_{N U(p-1) p} \\ G_{N U p 1} & \ldots & G_{N U p(p-1)} & 0\end{array}\right]+$

$\left[\begin{array}{cccc}S_{T U 1} & 0 & \ldots & 0 \\ 0 & S_{T U 2} & \ldots & \ldots \\ \ldots & \ldots & \ldots & 0 \\ 0 & 0 & 0 & S_{T U p}\end{array}\right] *\left[\begin{array}{cccc}0 & G_{F U 12} & \ldots & G_{F U 1 p} \\ G_{F U 21} & 0 & \ldots & \ldots \\ \ldots & \ldots & 0 & G_{F U(p-1) p} \\ G_{F U p 1} & \ldots & G_{F U p(p-1)} & 0\end{array}\right]$ 
Note: Due to the fact of invalidity commutative law for multiplying of matrixes, this sequence should be abided. On the other hand change of this obligatory sequence leads to same phenomenon as by changing disturbing and disturbed lines, called as crosstalk measurement, straight" and , across" or marked = and $x$.

\section{The Condition for Information Recoverability}

The couple of equations 1 and 2 is simplified for next consideration, again missing variable $\boldsymbol{\omega}$ for useful signal in downstream as well as upstream:

$$
\boldsymbol{S}_{\boldsymbol{R} i}=\boldsymbol{T}_{C T} \boldsymbol{S}_{T i} \quad \text { where } i=D, U
$$

and analogous noise circumstances in site of subscriber exchange:

$$
N_{R D}=S_{T D} G_{F D}+S_{T U} G_{N D}
$$

and again dually in site of network termination.

$$
N_{R U}=S_{T D} G_{N U}+S_{T U} G_{F U}
$$

The condition for information transmission secured in the simplest configuration as to Fig. 2 in sufficient quality may be performed for down(up)stream as follows:

$$
N_{R D(U) k}(\omega) \leq R_{D(U) k}(\omega) \quad \text { for } \omega \ni \Omega
$$

These inequalities may be overwritten for cable tree generally:

$$
\begin{aligned}
& N_{R D} \leq R_{D} \\
& N_{R U} \leq R_{U}
\end{aligned}
$$

Induction of both inequalities 8 into couple of equations 6 enables determination for highest assigned signals, there define masks of Power spectral density of transmitted signals:

$$
S_{T D} \leq-\frac{1}{G_{N U}-\frac{G_{F U} G_{F D}}{G_{F U}}}\left(R_{U}-\frac{G_{N U} R_{D}}{G_{N D}}\right) \text { for } \boldsymbol{\omega} \boldsymbol{\exists} \mathbf{\Omega}
$$

and 


$$
S_{T U} \leq \frac{1}{G_{N D}-\frac{G_{F U} G_{F D}}{G_{N U}}}\left(R_{D}-\frac{G_{F D} R_{U}}{G_{N U}}\right) \text { for } \boldsymbol{\ni} \boldsymbol{\Omega}
$$

Inequality system 9 may be altered by other constants produced by legislative measures of non-technical character. Matrixes of higher order are proposed to be calculated generally; therefore the volume of necessary calculations may exceed realizable measures.

\section{List of symbols:}

$\boldsymbol{G}_{\boldsymbol{F D}}$ - matrix of transmitting functions of FEXT in downstream

$\boldsymbol{G}_{\boldsymbol{F U}}$ - matrix of transmitting functions of FEXT in upstream

$\boldsymbol{G}_{\boldsymbol{N D}}$ - matrix of transmitting functions of NEXT in downstream

$\boldsymbol{G}_{\boldsymbol{N U}}$ - matrix of transmitting functions of NEXT in upstream

$\boldsymbol{N}_{\boldsymbol{R} \boldsymbol{D}}$ - noise spectrum vector in site of receivers in downstream

$\boldsymbol{N}_{\boldsymbol{R U}}$ - noise spectrum vector in site of receivers in upstream

$\boldsymbol{R}_{\boldsymbol{D}} \quad$ - receiver sensitivity vector in downstream

$\boldsymbol{R}_{\boldsymbol{U}} \quad$ - receiver sensitivity vector in upstream

$\boldsymbol{S}_{\boldsymbol{R} D}$ - signal spectrum vector in site of receivers in downstream

$\boldsymbol{S}_{\boldsymbol{R} U}$ - signal spectrum vector in site of receivers in upstream

$\boldsymbol{S}_{\boldsymbol{T D}}$ - signal spectrum vector in site of transmitters in downstream

$\boldsymbol{S}_{\boldsymbol{T U}}$ - signal spectrum vector in site of transmitters in upstream

$\boldsymbol{T}_{\boldsymbol{C T}}$ - transmitting functions vector of cable tree

$\boldsymbol{\omega}$ - radian frequency

$\boldsymbol{\Omega}$ - frequency interval (transmitted band)

\section{References}

[1] Křepelka, V.: Doctoral Theses, VUT FEKT ÚTKO, Brno, 2005

[2] Meninger, M.: Úvod do správy spektra metalické sítě,

Telekomunikace 1/03, Praha, ISSN 0040-2591 